\title{
Mars, The Nearest Habitable World - A Comprehensive Program For Future Mars Exploration
}

White Paper Submitted to the National Academies Planetary Science and Astrobiology Decadal Survey

By the

NASA Mars Architecture Strategy Working Group (MASWG)

8 July 2020

\section{Contact:}

Bruce Jakosky (MASWG Chair) University of Colorado at Boulder

bruce.jakosky@1asp.colorado.edu

Cell phone 303-817-4955

\section{Committee members/co-authors:}

Shane Byrne, U. Arizona

Wendy Calvin, U. Nevada Reno

Shannon Curry, UC Berkeley

Bethany Ehlmann, Caltech/JPL

Jen Eigenbrode, NASA/GSFC

Tori Hoehler, NASA/Ames

Briony Horgan, Purdue U.

Scott Hubbard, Stanford U.

Tom McCollom, CU/LASP

John Mustard, Brown U.

Than Putzig, Planetary Science Inst.

Michelle Rucker, NASA/JSC

Michael Wolff, Space Science Inst.

Robin Wordsworth, Harvard U.

\section{Ex Officio:}

Michael Meyer, NASA HQ

Rich Zurek, Mars Program Office, JPL 


\section{Introduction}

In response to a recommendation from the NASEM Planetary Science Mid-Term Decadal Survey Committee, NASA chartered MASWG to: (i) Determine what could and should be done in the scientific exploration of Mars beyond (i.e., in addition to or after) the Mars Sample Return campaign; (ii) survey the compelling science addressable by various classes of missions during the period 2020-2035, building on the science goals outlined in Vision \& Voyages and updated in the MEPAG Goals Document; (iii) define mission candidates in various mission classes to guide future Mars Exploration Program planning including, but not necessarily restricted to, missions in the small-spacecraft, Discovery, and New Frontiers categories, which may also be considered by the upcoming Planetary Decadal Survey (20232032); and (iv) define strategic technologies, infrastructure, and partnerships (international and commercial) that can enable compelling science in the specified time horizon, showing their programmatic linkage. MASWG carried out its study in fall 2019 and winter/spring 2020, presented preliminary recommendations to NASA HQ and to the Mars community in June 2020, and at this writing is in process of assembling its full written report and recommendations.

In summary:

- Mars has a uniquely accessible physical and chemical record of planetary processes spanning more than 4 billion years. It provides an unparalleled opportunity to study the climate, geology, geophysics, and habitability of a terrestrial planet.

- A distinct and identifiable Mars program within NASA's Science Mission Directorate, separately funded, is necessary to accomplish this compelling science and to help prepare for human missions. A program provides feed-forward on both science and technology development, coordination across missions to achieve the science objectives, coordination with international and corporate partners, and coordination with HEOMD to ensure that objectives necessary to support humans can be attained.

- A Mars program can most effectively address the full range of key science objectives by appropriately utilizing missions in all size classes, in addition to MSR. The key is to match the mission class to the science objectives, spanning the range from small spacecraft up through at least New-Frontiers-class missions.

- Four "mission arc" scenarios were defined in different high-priority science areas as examples to demonstrate how a cost-effective Mars Exploration Program could pursue compelling science objectives across a suite of missions over the next fifteen years.

\section{Science Rationale: Mars Is A Compelling Target For Both Science And Exploration}

Mars has a uniquely accessible archive of the long-term evolution of a habitable planet. The well-exposed 4-billion-year record of physical and chemical planetary processes is unique in the solar system because of its preservation, accessibility, and importance to understanding planetary habitability. This record includes planetary formation, impact bombardment, interior and crustal processes, atmospheric and climate evolution, and potentially the origin and evolution of life on another planet.

Mars presents outstanding access to environments fundamental to the search for past and/or present signs of life. Evidence for prebiotic chemical evolution and the origin of life has been almost entirely erased from Earth, but diverse geologic terrains from this era are preserved 
and accessible on Mars. Whether life ever existed or not on Mars will inform our understanding of the origin(s) of life on Earth and beyond.

Mars offers an unparalleled opportunity to study climate and habitability as an evolving, system-level phenomenon. The Martian climate has evolved dramatically through time, from an initial state with abundant liquid water to today's cold and dry surface. Mars exploration has shown us that habitability is a time-dependent phenomenon governed by interacting processes that occur over a range of spatial and temporal scales. The longevity and accessibility of Mars' rock and volatile record allows us to study how interior, atmospheric, and impact processes together drive climate and habitability, and their evolving nature. The present climate is observable directly, and the record of past climate is stored in the volatile deposits of the polar caps, the crustal rock record, and today's atmosphere; this ancient record has been largely lost on the Earth.

Mars is the best place in our solar system to study the first billion years of evolution of a habitable terrestrial planet. Access to relatively pristine terrains that record the end of planetary formation, the coupled early geophysical and geological history, the early evolution of an atmosphere, and the potential for an origin of life is unparalleled. These processes cannot be studied in the same way on the Earth or Venus due to the paucity of unaltered ancient materials, nor on the Moon or Mercury due to the absence of coupling with atmospheric evolution and habitability. Additionally, the ancient terrains on Mars are readily accessible to scientific study.

Outstanding opportunities for elucidating the climate, prebiotic and possible biological history of Mars informs our understanding of the evolution of exoplanets. Processes operating at Mars have operated and may be operating currently on many planets around other stars. Mars is the only place with that record where the relevant processes can be studied in detail. These fundamental problems of planetary evolution bring together our understanding of Earth, the terrestrial planets of our solar system, and beyond.

Finally, Mars is a compelling destination for human exploration and science/exploration synergies. After the Moon, Mars is the next-most-accessible destination for humans. Future human exploration and science investigations at Mars are complementary activities that can leverage advancements from each other. New science investigations (such as understanding the dust cycle and the formation of lower-latitude ice deposits) support planning of human exploration activities. In turn, the arrival of humans at Mars will dramatically enhance our ability to achieve big-picture science objectives.

\section{Why A Mars Program Is Needed}

Justification for Mars having a separate program has scientific, programmatic, and exploration elements:

Scientific: Mars provides the best opportunity to explore the full range of processes and properties on terrestrial planets under different boundary conditions from Earth, including interactions between geological, geophysical, climate/atmosphere, space weather, and potential biological processes. Unlike other planets in our solar system (including even the Earth), Mars' entire history is well preserved in an accessible rock record that includes the first billion years. Mars has key similarities with the Earth to allow us to understand the processes that operated, with enough differences to truly test our models and our understanding 
Programmatic: Mars is accessible enough to allow multiple missions to explore the different components of the Mars environment and their interactions with each other, including straightforward and long-lived access to the surface.

Exploration: Mars is NASA's stated long-term destination for human exploration.

Having a separate Mars program allows multiple necessary activities to be performed outside of any single mission:

- Allows coordination and continuity between missions to achieve science objectives beyond what a single mission or even a series of one-off missions could accomplish.

- Provides feed-forward between missions on both science and technology, including use of small spacecraft as proof of concept for innovative approaches or measurements.

- Allows development of infrastructure that can serve multiple missions (e.g., communications relay from orbit, landing-site selection).

- Allows effective negotiation and coordination with international and commercial partners to take advantage of the tremendous global interest in exploring Mars.

- Allows focused development of required spacecraft and instrument technology in advance of mission selection (e.g., Mars Ascent Vehicle (MAV) development for MSR).

- Allows coordination with HEOMD to ensure strong connections between the human and robotic programs for Mars.

\section{MASWG High-Level Recommendations}

1. Mars Sample Return should proceed as currently planned, as it will produce a major step forward in our understanding of Mars, as envisioned by Visions \& Voyages.

2. NASA should support missions that address fundamental science objectives at Mars in addition to MSR, using the full range of technically viable mission classes. During the MSR era, the emphasis should be on achieving other high-priority science objectives, while developing the needed technologies for going forward.

3. To the extent possible, missions and instruments should be openly competed; where specific investigations are desired, objectives can be defined and then opened to competition.

4. A robust Mars exploration program will require affordable access to multiple places on the Martian surface and affordable long-lived orbiters. NASA should invest early to expedite the rapidly evolving small spacecraft technologies and procedures to achieve these capabilities at lower costs than past missions.

5. For this next phase of Mars exploration, NASA should retain a programmatically distinct Mars Exploration Program within the Science Mission Directorate. NASA should institute mission or budget lines that can allow Mars-specific missions, from small spacecraft through New Frontiers-class missions, to be strategically integrated into a program, with missions chosen and implemented as appropriate for the science to be achieved.

6. The relationship between the SMD Mars Exploration Program (traditionally referred to as the "robotic program") and HEOMD campaigns ("human exploration") needs to be further developed. The SMD MEP can provide information and infrastructure needed to enable exploration by humans on Mars in a safe and cost-effective way and can identify the science that such human exploration should pursue when humans are on the planet.

\section{MASWG Recommendations for a Successful Future Mars Exploration Program}


MASWG makes the following recommendations for implementation of a successful Mars Exploration Program:

1. The guiding principles required to drive the program should include:

- Address science priorities as defined by the Decadal Survey and by MEPAG;

- Be responsive to discoveries by ongoing and new missions;

- Have missions build on each other both scientifically and technologically;

- Compete missions or payload elements to the extent possible within strategic direction;

- Inject a sufficient number of flight opportunities to sustain technical capability and to achieve steady progress on key scientific goals; frequent missions may be essential to attracting the commercial sector and international partners;

- The choice of mission class should be determined by the specific science objectives.

2. Program should be sustained at a steady funding level, with commensurate results. The size and scope of the program - and therefore the progress that can be achieved - will depend upon the resources provided.

3. Within the Program, NASA should develop a line of PI-led small spacecraft, Discovery and New Frontiers-class missions, competed in a separate program line while addressing strategic goals.

4. The Program should have a protected, adequately funded, and competed technology development program to advance instrumentation and developments in key areas (e.g., as is being done for the MAV). The technology invested should be focused and leveraged within NASA and with other agency and commercial entities.

5. NASA should develop low-cost approaches for entry vehicles at all size classes, including entry, descent, and landing; for long-lived orbiting spacecraft; and for aerial vehicles, landers, and rovers to provide access and mobility after landing.

6. NASA and the Mars community should study the feasibility of adapting the CLPS program to Mars. A successful Mars-focused Commercial Mars Payload Services (CoMPS) could serve as a programmatic vehicle to allow, at reduced cost, development of technologies for future exploration as well as delivery of science payloads.

7. NASA and the Mars community should continue to explore, negotiate, and support international collaborations as a means of leveraging flight opportunities to achieve compelling science.

- Involve the respective scientific communities in the definition and execution of joint missions;

- To the extent possible, compete missions and instruments to get the best science;

- Financially support the mission participants adequately to achieve the mission objectives (Instrument Teams, Science Team members, Participating Scientists, Interdisciplinary Scientists).

8. NASA should adequately fund the analysis of returned mission data to maximize the scientific return on mission investment and ensure that results can be achieved in timely fashion; extended missions should be supported as long as they make solid scientific progress.

9. NASA needs to enhance interactions between the revitalized Mars Exploration Program in SMD and the Human Exploration \& Operations Mission Directorate (HEOMD) to define needs and the opportunities to address them. Such interaction would ensure that: 
- Adequate, accurate, and appropriate information and experience is provided in support of human missions;

- Scientific progress is sustained and advanced by missions with humans.

\section{Possible Mission Architectures}

We developed four different prototype science arcs to explore how coordinated sets of missions could be integrated to maximize scientific progress and examine the potential role of missions in different cost/size classes. The four examples demonstrate that a program can be constructed addressing compelling science arcs. There are other potential "arcs"; the ones cited here are meant only to demonstrate that such strategically linked, compelling arcs exist. While the examples were chosen because they address what MASWG believes are high-priority science objectives, the final definition of the future mission architecture should be the result of the program working with the scientific community. Progress along multiple arcs is possible in the next decade, in some cases with the same choice of missions, and the scope of what can be accomplished depends on adequate funding.

In most cases there is a progression (phases) from building on what is known today (e.g., diverse environments) and with existing capabilities to more-capable missions, often after the currently proposed next MSR flight elements are well along. The increased capability is typically driven by the payload as more-complex measurements are needed to follow up earlier discoveries or more-challenging science objectives. (An exception might be the long-term observations of the current climate where a succession of small satellites with a standardized payload, modestly upgraded over time, could achieve much of that goal.)

While individual missions in a mission arc could be achieved through the Discovery/New Frontiers competitive process, inclusion in a strategic program line would ensure a consistent approach with missions building on one another. (New Frontiers should not continue to be closed to Mars missions if missions of that class are prohibited by inadequate funding within this new Mars program.)

The four mission arc examples (detailed in upcoming full report) that were examined are:

1. Diverse habitable environments. Explore diversity of ancient Mars, following up on the thousands of possible sites identified in orbital data, to understand early planetary evolution and the nature, origin, and timing of environments, habitability, and/or biological potential of Mars.

2. Subsurface structure, composition and life. The subsurface of Mars is largely unexplored and yet its structure and composition holds many clues to the early evolution of Mars. Further, it could be the final refuge of an early Martian biosphere.

3. Recent climate change as recorded in ice. Understand Martian ice ages in terms of the distribution and stratification of ice as it was emplaced/removed over the last hundred million years, both in the polar regions and in lower latitudes.

4. Climate variability and process. Record variability of the current climate from hours to decades and the processes of transport and photochemistry, Sun-Mars interactions, exchange of water, dust, $\mathrm{CO}_{2}$ and trace gases.

The missions populating these four arcs were grouped into mission classes (with rough life-cycle cost ranges) of Small Spacecraft (SSc: $\$ 100-300 \mathrm{M}$ ), Discovery (DSc: $\$ 600-\$ 1000 \mathrm{M}$ ), New Frontiers (NFc: $\$ 1000-\$ 1500 \mathrm{M}$ ), and Flagship (FLG: $>\$ 1500 \mathrm{M})$. These costs are meant to include launch vehicles/ride accommodation and Phase E funding. 
Small spacecraft have great potential for carrying out significant Mars science. However, while orbital small spacecraft are viable today, critical technology maturation and development are needed to land meaningful payloads successfully onto the surface at an affordable cost. More-capable missions (Discovery, New Frontiers, Flagships after MSR) would be needed to accomplish the most challenging objectives and discoveries. The means by which commercial entities could provide key services (e.g., transport or communications) and to what extent and on what schedule needs to be investigated and developed; this could be critical to enabling specific science missions in several of the possible mission arcs.

A compelling Mars Exploration Program should pursue two or more mission arcs (these or others) in parallel with Mars Sample Return, dependent on the available funding in a dedicated, separate line for that purpose. Costs have the potential to be shared through both international and commercial collaborations.

A Mars program could achieve key science goals utilizing missions that span the entire range of cost levels. Figure 1 shows an example of the different types of science that can be accomplished with the different mission classes. Most importantly, we need to ensure that each mission class utilized is appropriate for the specific science goals and that the program is designed around the most critical science questions rather than which questions can be answered utilizing a given size class.

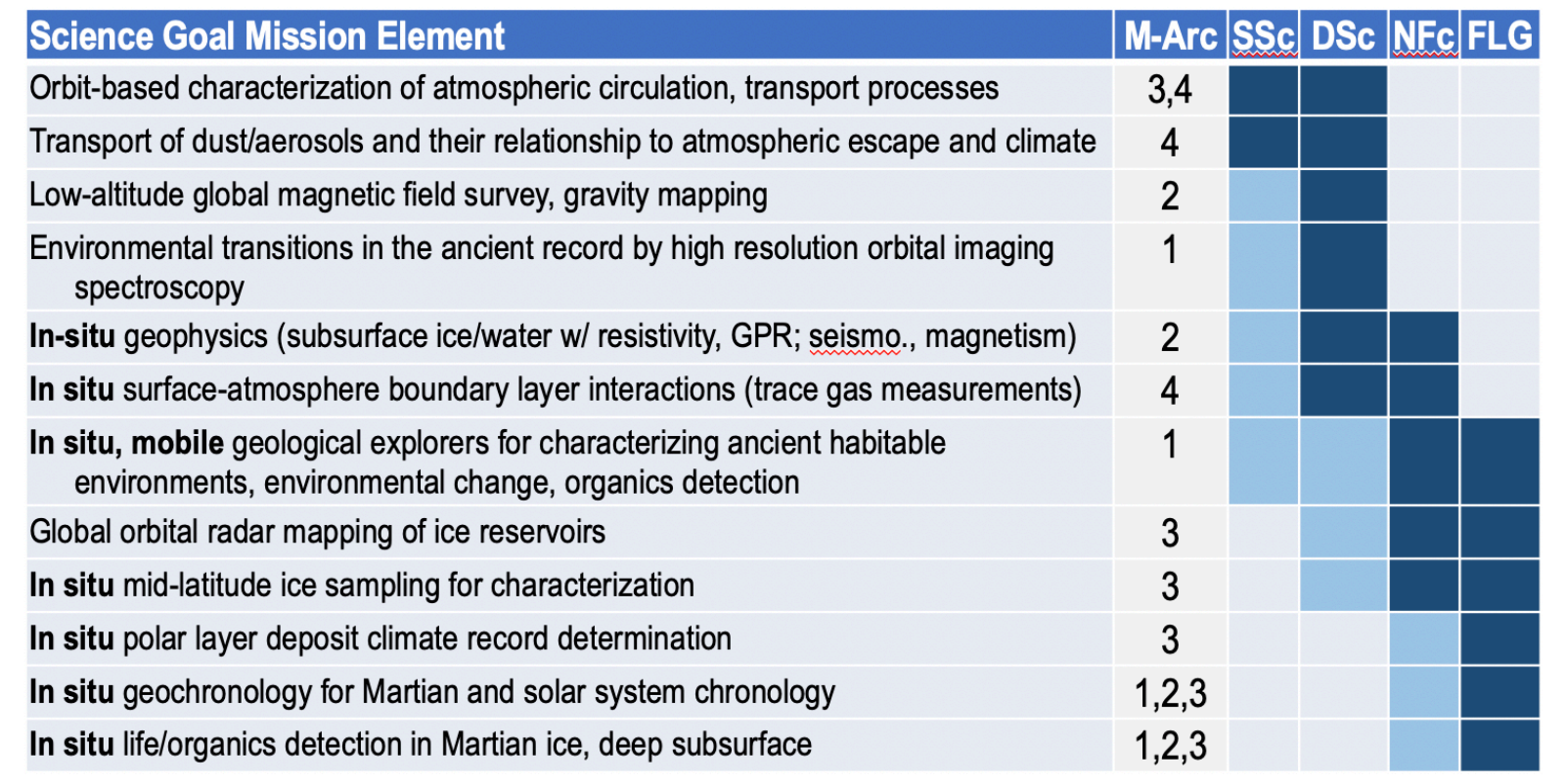

possible or partial priority science at this class

achieves priority science at this class

Figure 1. Example science objectives that can be addressed with different mission size classes. Mission arc designations are tied to the four arcs listed above.

Although we haven't attempted to determine realistic life-cycle costs or year-by-year cost profiles, rough estimates of the cost of the potential program that we've outlined can be made (Figure 2). Note that (i) a viable program should pursue elements of at least two mission arcs, (ii) there is no prioritization intended here between the mission arcs or against other possible arcs, (iii) costs do not reflect possible international contributions or commercial partnerships, (iv) lower costs before 2030 reflect accommodation of Mars Sample Return while building to post- 
2031 flights, and (v) flagship-class mission options to launch after MSR are likely to emerge, based on compelling science objectives or fundamental new discoveries. The final row of Figure 2 is a reminder that technology maturation, infrastructure (e.g., telecommunications or site characterization/certification), international participation in non-NASA missions, and extended mission costs need to be included and budgeted appropriately.

\section{Implementing a Mars Exploration Program}

In summary:

- Compelling scientific, programmatic, and technology arguments exist for NASA to support a vigorous and robust Mars program that operates in addition to and in parallel with the Mars Sample Return campaign.

- A Mars Exploration Program would take place most effectively and efficiently if it continued to operate separately and independently within the Planetary Sciences Division.

- Opportunities should be sought for both international and commercial collaborations that can enhance the Mars program, while utilizing competition for missions, payloads and instruments wherever possible.

- Small spacecraft have great potential for carrying out significant Mars science, with the added excitement of being able to have a launch at nearly every opportunity every two years.

- Rapid development in small spacecraft capabilities for Mars may lead to lower costs than estimated here.

- A critical development need is the ability to land capable small spacecraft on Mars at an affordable cost.

- More-capable missions (Discovery, New Frontiers, Flagships after MSR) will be needed to address the most challenging objectives and discoveries. All would benefit from lower-cost access to the surface.

- We presented several example mission arcs involving a variety of mission types that could make substantial scientific return in a Mars program; ultimately, the rate and extent of progress depends on the available funds.

\begin{tabular}{|c|c|c|c|}
\hline Mission Arc & $2021-2030$ & $2031-2035$ & Key Tech \\
\hline Arc 1: Diverse Habitable Envs. & $4 \mathrm{SSc}$ & $1 \mathrm{NFC}^{*}, 1 \mathrm{SSc}$ & Small Landers \\
\hline Arc 2: Subsurface & $1 \mathrm{SSc}, 1 \mathrm{DSc}$ & $1 \mathrm{NFc}^{*}$ & Drilling/Analysis \\
\hline Arc 3: Ice Science & $1 \mathrm{SSc}, 1 \mathrm{DSc}$ & $1 \mathrm{NFc}^{*}$ & Ice Landers \\
\hline $\begin{array}{l}\text { Arc 4: Climate Variability and } \\
\text { Processes }\end{array}$ & 2 ssc & $1 \mathrm{SSc}, 1 \mathrm{DSc}$ & $\begin{array}{l}\text { Network Landers } \\
\text { Long-lived SSc }\end{array}$ \\
\hline Assuming Progress on all Arcs & $\begin{array}{c}\sim \$ 300 \mathrm{M} / \mathrm{Xr} \\
(8 \mathrm{SSC}, 2 \mathrm{DSc}) \\
\end{array}$ & $\begin{array}{c}\sim \$ 500 \mathrm{M} / \mathrm{xc} \\
(2 \mathrm{ssc}, 1 \mathrm{DSc}, \text { *just } 1 \mathrm{NEc}) \\
\end{array}$ & \\
\hline Assuming Progress in $\sim 2$ Arcs & 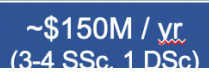 & 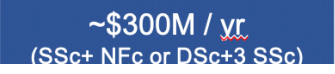 & \\
\hline
\end{tabular}

Figure 2. Possible mission queues and per-year cost estimates for each of the four mission arcs outlined above.

Acknowledgements: Funding for this activity was provided by the National Aeronautics and Space Administration. 